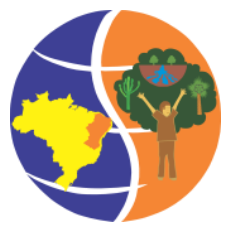

III Simpósio Brasileiro de Recursos Naturais do Semiárido - SBRNS

"Manejo de bacias hidrográficas em regiões semiáridas: potencialização da produção de água"

Fortaleza - Ceará, Brasil

20 a 22 de junho de 2017

doi: 10.18068/IIISBRNS2017.geoap736

ISSN: $2359-2028$

\title{
EVAPOTRANSPIRAÇÃO NO SEMIÁRIDO BRASILEIRO DETERMINADA COM PRODUTOS MODIS E ESTAÇÕES AGROMETEOROLÓGICAS
}

\author{
Antônio Heriberto de Castro Teixeira ${ }^{1}$, Janice Freitas Leivas ${ }^{2}$, Gustavo Bayma-Silva ${ }^{3}$, Edlene \\ Aparecida Monteiro Garçon ${ }^{4}$
}

\footnotetext{
${ }^{1}$ Ph.D., Embrapa Monitoramento por Satélite, Campinas-SP, 19 32116200, heriberto.teixeira@embrapa.br

2 Dr., Embrapa Monitoramento por Satélite, Campinas-SP, janice.leivas@embrapa.br

${ }^{3}$ MSc., Embrapa Monitoramento por Satélite, Campinas-SP, gustavo.bayma@embrapa.br

${ }^{4}$ BSc., Embrapa Monitoramento por Satélite, Campinas-SP, edlene.garcon@embrapa.br
}

RESUMO: Objetivando subsidiar o manejo racional dos recursos naturais na região semiárida do Nordeste do Brasil, imagens MODIS ao longo do ano de 2016 foram usadas em conjunto com uma rede de estações agrometeorológicas. $\mathrm{O}$ algoritmo SAFER foi aplicado para modelagem da evapotranspiração atual (ET) nos municípios de Petrolina-PE e JuazeiroBA. Claramente pôde-se ver valores mais elevados da ET para culturas irrigadas (CI) que para vegetação natural $(\mathrm{VN})$ em ambos os municípios. Apesar dos municípios terem apresentado uma dinâmica similar nas taxas evapotranspiratórias ao longo do ano, em Juazeiro, estas representaram $78 \%$ daquelas em Petrolina. Uma razão para os valores mais baixos em Juazeiro pode ser devida aos menores consumos da água de irrigação pelas culturas agrícolas. PALAVRAS-CHAVE: sensoriamento remoto, recursos hídricos, SAFER

\section{EVAPOTRANSPIRATION IN THE BRAZILIAN SEMI-ARID BY USING MODIS PRODUCT AND AGROMETEOROLOGICAL STATIONS}

\begin{abstract}
Aiming subsidizing the rational water resources management in the semi-arid region of the Northeast Brazil, MODIS images along the year 2016 were used together with a net of agrometeorological stations. The SAFER algorithm was applied for modelling actual evapotranspiration (ET) in the Petrolina-PE and Juazeiro-BA counties. Clearly one could see higher ET values for irrigated crops (IC) than for natural vegetation (NV) in both counties. Even the counties presenting similar evapotranspiration rates dynamics along the year, in Juazeiro they represented $78 \%$ of those for Petrolina. One reason for the smaller ET values in Juazeiro could be because to the lower irrigation water consumption by crops.
\end{abstract}

KEYWORDS: remote sensing, water resources, SAFER

\section{INTRODUÇÃO}

Os municípios de Petrolina-PE e Juazeiro-BA, nas condições semiáridas do Nordeste do Brasil, são exemplos de crescimento agrícola, devido ao desenvolvimento das tecnologias de irrigação aplicadas nas áreas vizinhas do Rio São Francisco. Sob estas condições de rápida mudança de uso da terra, o uso de geotecnologias é relevante (Teixeira et al., 2015).

O uso da equação de Penman-Monteith (PM) tem sido sugerido para aplicação em conjunto com parâmetros obtidos por sensoriamento remoto, tanto na obtenção da ET como para o coeficiente de cultura $\left(\mathrm{K}_{\mathrm{c}}\right)$. Este último coeficiente é a razão da ET para a 
evapotranspiração de referência $\left(\mathrm{ET}_{0}\right)$ em boas condições de umidade na zona das raízes das plantas (Kamble et al., 2013), porém os baixos valores da razão ET/ET 0 indicam condições de estresse hídrico (Lu et al.,2011). Baseado na modelagem, da razão ET/ET 0 com parâmetros obtidos por sensoriamento remoto, o SAFER (Simple Algorithm for Evapotranspiration Retrieving) foi desenvolvido, validado e aplicado para a obtenção da ET e do $\mathrm{K}_{\mathrm{c}}$ em diferentes condições ambientais brasileiras (Teixeira et al., 2015).

O objetivo do atual trabalho é a aplicação do SAFER em conjunto com uma rede de estações agrometeorológicas, cobrindo diferentes condições termo hídricas ao longo do ano de 2016, para a determinação da ET em agros-ecossistemas dos municípios de Petrolina, estado de Pernambuco (PE) e Juazeiro, estado da Bahia (BA).

\section{MATERIAL E MÉTODOS}

A Figura 1 apresenta a localização das estações agrometeorológicas utilizadas em conjunto com o produto MODIS MOD13Q1 nos municípios de Petrolina, estado de Pernambuco (PE) e Juazeiro, estado da Bahia (BA), na região semiárida do Nordeste do Brasil.

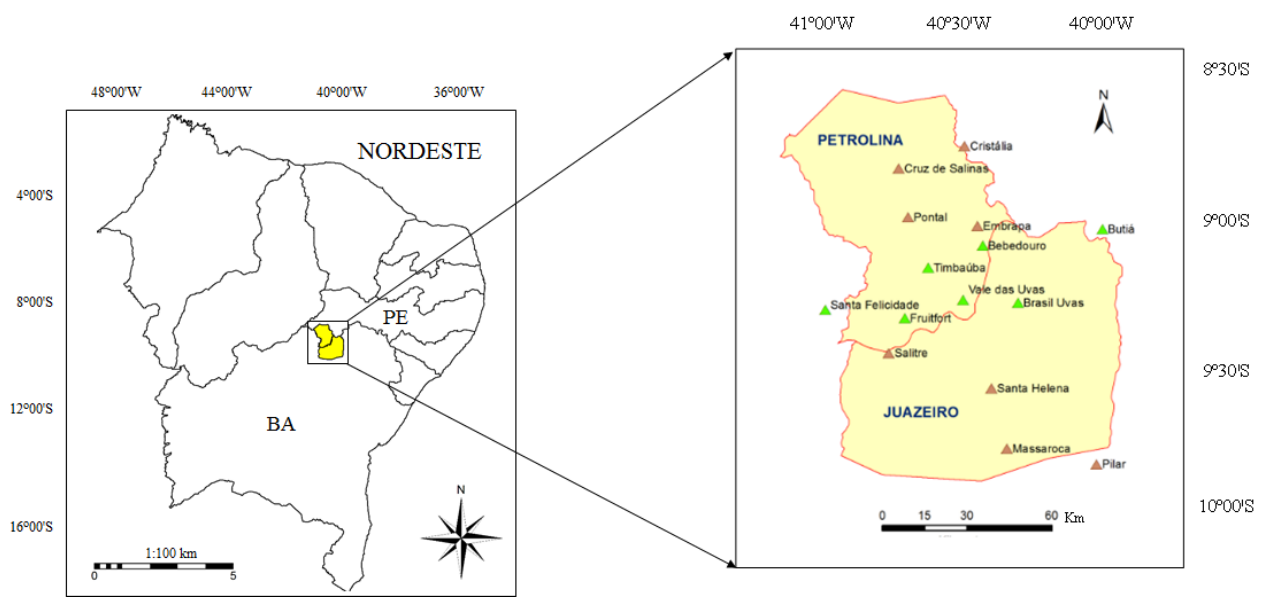

Figura 1. Localização das estações agrometeorológicas nos municípios na de Petrolina-PE e Juazeiro-BA, Nordeste do Brasil. Triângulos verdes simbolizam as estações dentro de fazendas irrigadas, enquanto que os marrons indicam aquelas em áreas de Caatinga.

O produto MOD13Q1 utilizado fornece um total de 23 imagens das reflectâncias nas bandas 1 e 2 ao longo do ano, as quais em conjunto com dados climáticos, possibilitaram a obtenção da ET sob condições termo hídricas ao longo do ano de 2016, na escala temporal de 16 dias (Teixeira et al., 2015). 
O algoritmo SAFER foi usado para modelar a razão da ET para a $\mathrm{ET}_{0}$, a $\mathrm{ET}_{\mathrm{r}}$ :

$\mathrm{ET}_{\mathrm{r}}=\exp \left[\mathrm{a}+\mathrm{b}\left(\frac{\mathrm{T}_{0}}{\alpha_{0} \mathrm{NDVI}}\right)\right]$

Em que $\alpha_{0}$ - é o albedo da superfície; NDVI - é o Índice da Diferença de Vegetação Normalizado, ambos calculados com as bandas 1 e 2; $\mathrm{T}_{0}$ - é a temperatura da superfície estimada por resíduo no balanço de radiação, e a e b são coeficientes de regressão. Detalhes da obtenção destes parâmetros são encontrados em Teixeira et al. (2015).

$\mathrm{A} \mathrm{ET}_{\mathrm{r}}$ é então multiplicada pelas grades diárias de $\mathrm{ET}_{0}$ fornecendo os valores da ET para 24 horas (Teixeira et al., 2015):

$\mathrm{ET}=\mathrm{ET}_{\mathrm{r}} \mathrm{ET}_{0}$,

\section{RESULTADOS E DISCUSSÃO}

A Figura 2 apresenta as tendências dos totais médios da precipitação $(\mathrm{P})$ e da $\mathrm{ET}_{0}$ para os períodos de 16 dias das imagens MODIS nos municípios de Petrolina-PE e Juazeiro-BA, durante o ano de 2016.

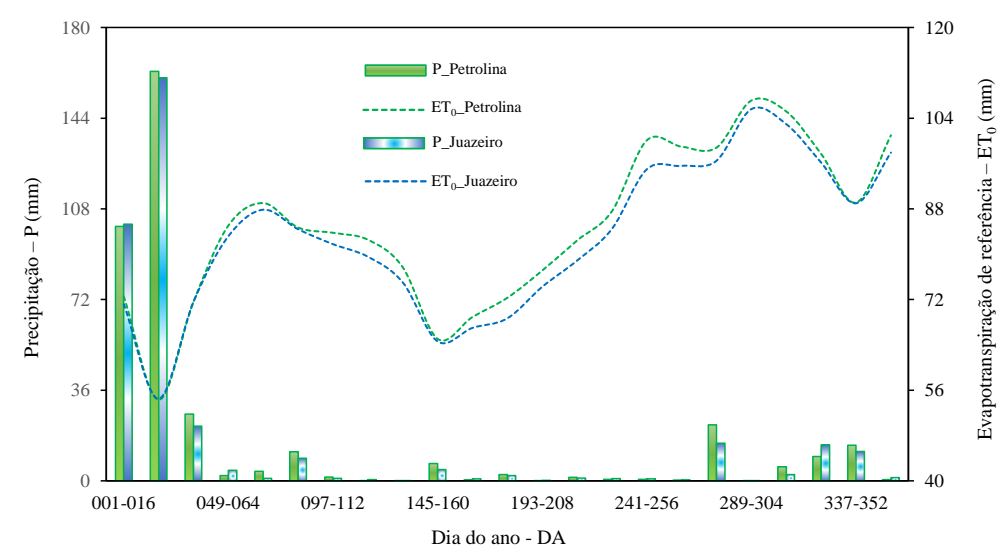

Figura 2. Componentes do balanço hídrico climático nos municípios de Petrolina-PE e Juazeiro-BA, para intervalos de 16 dias das imagens MODIS: P - Precipitação; ET $_{0}$ Evapotranspiração de referência.

A precipitação (P) foi mais variável que a $\mathrm{ET}_{0}$ ao longo do ano, com as chuvas se concentrando no mês de janeiro (DA 001 a 032) quando chegou a ser o triplo de $\mathrm{ET}_{0}$ na segunda quinzena deste mês, em ambos os municípios. Entretanto, após este mês percebe-se carência de chuvas com várias situações com $\mathrm{P}$ próximo de $0 \%$ da $\mathrm{ET}_{0}$, indicando fortes condições de escassez hídrica ao longo do ano. 
Os valores mais elevados da $\mathrm{ET}_{0}$ ocorreram no início e no final do ano de 2016, acima de $80 \mathrm{~mm}$ para os períodos de 16 dias. Apesar do total anual de chuvas em Petrolina $(\mathrm{P}=374$ $\mathrm{mm}$ ) ter sido superior ao de Juazeiro $(\mathrm{P}=355 \mathrm{~mm})$, com uma demanda atmosférica maior no primeiro município $\left(\mathrm{ET}_{0}=1952 \mathrm{~mm}\right)$ que no segundo $\left(\mathrm{ET}_{0}=1905\right)$, as quantidades de chuvas em ambos atenderam apenas 19\% da demanda atmosférica, mostrando que a irrigação é indispensável para a produção de alimentos na região, porém deve ser realizada de maneira racional, baseada nos requerimentos hídricos das culturas, os quais exigem o conhecimento da ET nos diferentes agros-ecossistemas.

A Figura 3 apresenta a distribuição espacial dos valores médios diários da ET, para períodos específicos de 16 dias do produto MOD13Q1, abrangendo diferentes condições termo hídricas ao longo do ano de 2016, nos municípios de Petrolina-PE e Juazeiro-BA, Nordeste do Brasil.

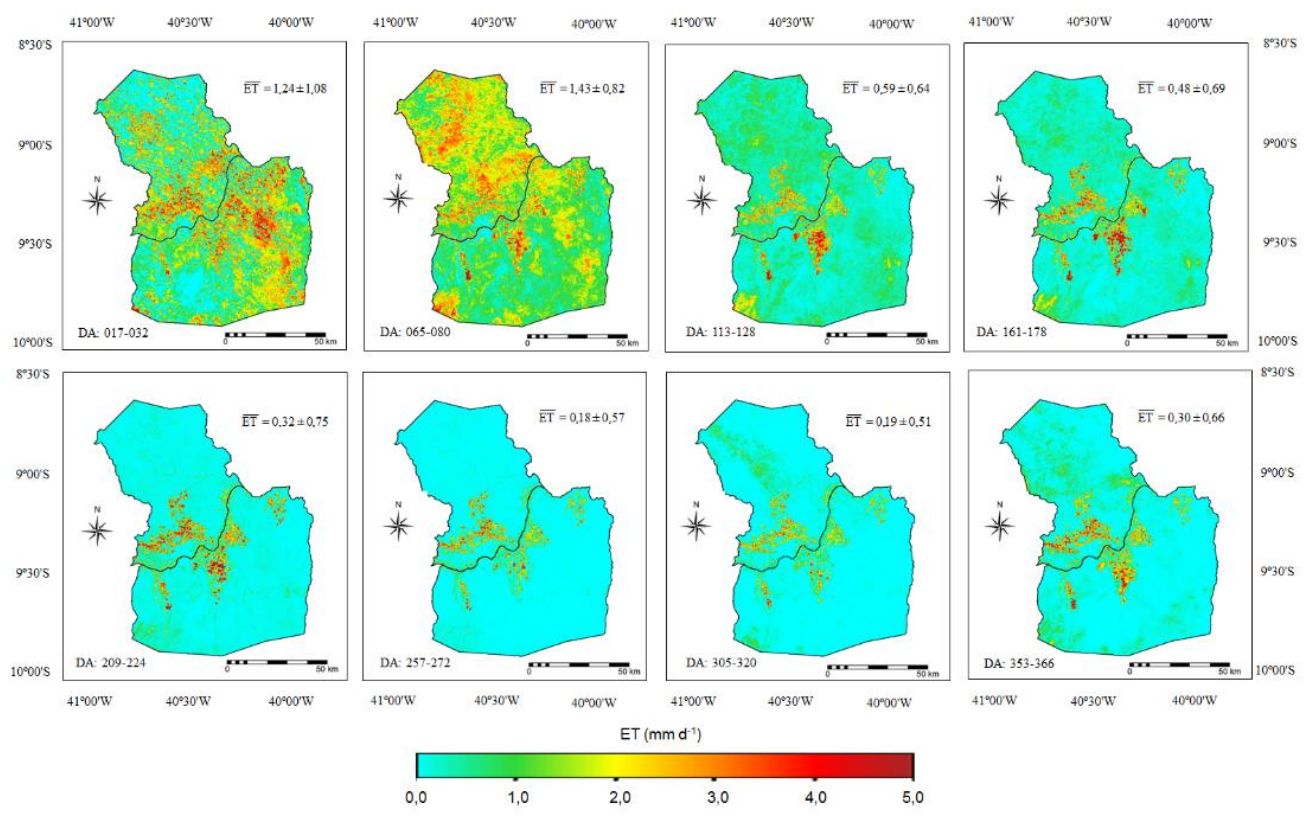

Figura 3. Distribuição espacial dos valores médios diários da evapotranspiração atual (ET) para períodos específicos de 16 dias do produto MOD13Q1, abrangendo diferentes condições termo hídricas do ano de 2016, nos municípios de Petrolina-PE e Juazeiro-BA.

As variações espaciais e sazonais da ET ao longo do ano de 2016 são claras comparando-se os períodos chuvosos com aqueles mais secos (ver Figuras 2 e 3), confirmando a sensibilidade do SAFER para detectar as diferentes condições hídricas e de vegetação. Com exceção dos primeiros meses do ano quando ocorreram chuvas abundantes, claramente pode-se distinguir as culturas irrigadas nas vizinhanças do Rio São Francisco das 
espécies da Caatinga pelos valores da ET mais elevados no primeiro ecossistema. Os pixels com médias em torno de $5 \mathrm{~mm} \mathrm{~d}^{-1}$ representam principalmente fruteiras bem irrigadas.

Santos et al. (2010), aplicando os algoritmos SEBAL e S-SEBS com imagens Landsat, reportaram valores máximos de ET de $6,0 \mathrm{~mm} \mathrm{~d}^{-1}$ em agros-ecossistemas nas condições do semiárido brasileiro. Também na região semiárida do Brasil, mas neste caso usando o modelo METRIC, Folhes et al. (2009) encontraram valores mais baixos para Caatinga $\left(<2,0 \mathrm{~mm} \mathrm{~d}^{-1}\right)$, valores moderados para fruteiras $\left(3,0\right.$ a $\left.6,0 \mathrm{~mm} \mathrm{~d}^{-1}\right)$, similares ao atual estudo. A maior razão para os valores mais elevados para a Caatinga no atual estudo no início do ano se deve ao fato das imagens estarem dentro do período chuvoso, fazendo com que as espécies naturais tenham taxas evapotranspiratórias similares àquelas das culturas irrigadas.

A Figura 4 apresenta os valores médios e desvios padrões da ET para cada período de 16 dias do produto MOD13Q1 envolvendo as diferentes condições ao longo do ano de 2016 nos municípios de Petrolina-PE e Juazeiro-BA.

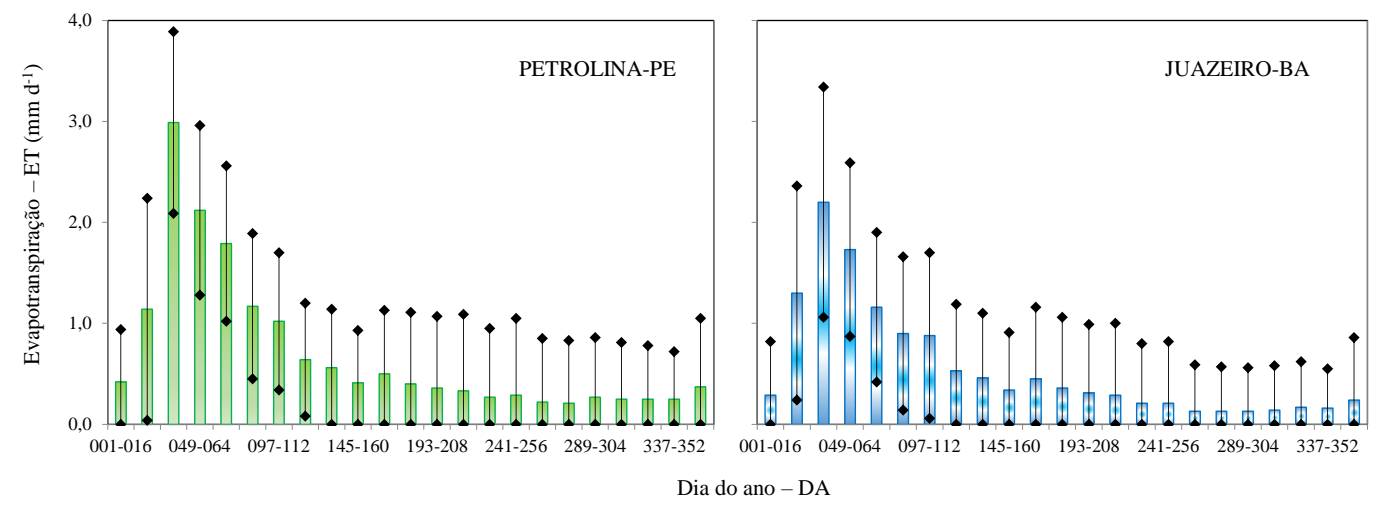

Figura 4. Valores médios diários e desvios padrões da evapotranspiração atual (ET) nos diferentes agros-ecossistemas dos municípios de Petrolina-PE e Juazeiro-BA, para cada período de 16 dias do produto MOD13Q1.

Para ambos os municípios, as maiores taxas evapotranspiratórias foram da segunda quinzena de janeiro até a metade de abril. Neste período, considerando-se os valores superiores dos desvios padrões, a ET para as culturas chegou a valores médios de 4,0 $\mathrm{mm}$ d-1, no período de DA 033-049 (fevereiro), representando o efeito conjunto das chuvas e irrigação, enquanto que após o DA 128, na primeira quinzena de maio, grande parte das espécies da Caatinga restringiram a transpiração, como consequência da redução/ausência das chuvas sob condições de escassez hídrica severa (ver também Figura 2). 
Apesar de Petrolina e Juazeiro terem apresentado uma dinâmica similar nas taxas evapotranspiratórias ao longo do ano de 2016, estas taxas no segundo município representaram $78 \%$ daquelas do primeiro. Mesmo com menores quantidades de chuvas em Juazeiro, estas foram compensadas por uma demanda atmosférica mais baixa, com $\mathrm{P}$ atendendo $19 \%$ da $\mathrm{ET}_{0}$ em ambos os municípios. Então uma razão para os menores valores da ET em Juazeiro pode ser devida aos menores consumos da água de irrigação proveniente do Rio São Francisco pelas culturas agrícolas.

\section{CONCLUSÕES}

A evapotranspiração em larga escala foi modelada pela união do produto MODIS MOD13Q1 e dados de estações agrometeorológicas nas condições semiáridas nos municípios de Petrolina-PE e Juazeiro-BA, com aplicação do algoritmo SAFER. Esta combinação é útil para o monitoramento da dinâmica das condições hídricas e de vegetação nas condições semiáridas do Nordeste do Brasil no ano de 2016. Juazeiro apresentou uma menor quantidade de chuvas que Petrolina, porém com demanda atmosférica inferiores, com taxas evapotranspiratórias mais baixas resultantes de um menor consumo de água de irrigação proveniente do Rio São Francisco.

\section{REFERÊNCIAS}

FOLHES, M.T.; RENNÓ, C.D.; SOARES, J.V. Remote sensing for irrigation water management in the semi-arid Northeast of Brazil. Agricultural Water Management, v. 96, p. 1398-1408, 2009.

SANTOS, C.A.C. dos; BEZERRA, B.G.; SILVA, B.B. da; RAO, T.V.R. Assessment of daily evapotranspiration with SEBAL and S-SEBI algorithms in cotton crop. Revista Brasileira de Meteorologia, v. 25, p. 383-392, 2010.

KAMBLE, B.; KILIC A.; HUBARD, K. Estimating Crop Coefficients Using Remote Sensing-Based Vegetation Index. Remote Sensing, v. 5, p. 1588-1602, 2013.

LU, N.; CHEN, S.; WILSKE, B.; SUN, G.; CHEN, J. Evapotranspiration and soil water relationships in a range of disturbed and undisturbed ecosystems in the semi-arid Inner Mongolia, China. Journal of Plant Ecolology, v. 4, p. 49-60, 2011.

TEIXEIRA, A.H. de C.; HERNANDEZ, F.B.T.; SCHERER-WARREN, M.; ANDRADE, R.G; LEIVAS, J.F.; VICTORIA, D. de C.; BOLFE, E.L.; THENKABAIL, P.S.; FRANCO, R.A.M. Water Productivity Studies from Earth Observation Data: Characterization, Modeling, and Mapping Water Use and Water Productivity. In: PRASAD, S.T. (Ed.). Remote Sensing of Water Resources, Disasters, and Urban Studies. 1ed.Boca Raton, Florida: CRC Group, Taylor and Francis, p. 101-126, 2015. 